\title{
Effects of overt rehearsal on recall of paired associates*
}

\author{
STEPHEN MADIGAN \\ University of Southern California \\ Los Angeles, California 90007
}

It has been reported that overt rehearsal of lists eliminates negative recency effects in free recall (Atkinson \& Shiffrin, 1971). However, in probe paired-associates tests, overt rehearsal appears only to lower recall levels, relative to "silent" rehearsal conditions; the extent of negative recency effects is unaltered.

This note describes the effects of an overt rehearsal procedure on immediate and delayed recall of paired associates. Rundus (1971) has reported a variety of effects that are obtained when Ss are required to rehearse material aloud during presentation of to-be-remembered lists of words. One such effect is modification of the serial position curve of free recall. A one-element rehearsal set that requires $S$ to repeat items one at a time eliminates the primacy effect in immediate free recall (Atkinson \& Shiffrin, 1971). Presumably, the primacy effect arises with uncontrolled rehearsal when S groups or unitizes the first few elements in a list, aided by a low storage load (or an empty processing buffer) at the beginning of list presentation (Tzeng, Snyder, \& Hung, 1972). Similarly, a second effect of a one-item overt rehearsal is the elimination of the negative recency effect (Atkinson \& Shiffrin, 1971; Tzeng et al, 1972). This result seems to be implied by a selective rehearsal interpretation of the negative recency effect: equating number of rehearsals over all list positions should yield a flat serial position curve in a delayed recall test, without depressed recall of the last few input positions.

The experiment described in this paper was an application of an overt rehearsal procedure to a probe paired-associates task, and the comparison of this method with "silent" rehearsal conditions in terms of the relative extents of the negative recency effect they produce. In the absence of pair-by-pair rehearsal requirements, the PA probe test produces very large negative recency effects, with, typically, very few of the pairs from the last input position of lists being recalled in a delayed or final recall test (Madigan \& McCabe, 1971). It will be seen that, unlike its effect on free recall, the overt rehearsal procedure does nothing to lessen these effects; it produces only lower recall levels overall.

\section{METHOD AND PROCEDURE}

The basic test was a PA probe task, using auditory presentation of five-pair lists, with one pair presented every $3 \mathrm{sec}$. The five probe positions were tested in a random sequence. Thirty-two Ss performed this test with "silent" rehearsal; another 32 Ss were required to repeat each pair aloud once as soon as it was presented. Each $S$ was tested with a sequence of 25 lists, with each probe position (1-5) tested five times in a random order. Ss wrote their

\footnotetext{
*This research was supported by National Science Foundation
} Grant GB-36172. responses on cards, and there was a 10-sec interval between lists.

The final recall test involved a series of 50 probe tests. Twenty-five of these were for previously tested pairs; the other 25 probes were for previously presented pairs that had not been tested in immediate recall. These pairs were selected at random from each of the 25 lists, with constraints to produce 5 such tests for each probe position. The ordering of the 50 final tests was randomly determined for each $\mathrm{S}$. This test was unpaced, with the $\mathrm{S}$ proceeding through a 50-card deck of punch cards, each one of which displayed one probe item. Thus, the original presentation was auditory, but the final test was visual. (This shift of mode seems to have no effect on recall levels or their interaction with other experimental variables.) Two independent replications of materials were used.

\section{RESULTS AND DISCUSSION}

All of the immediate and final recall data are shown in Table 1 . Each entry is based on 160 observations ( 32 Ss by 5 tests) for each rehearsal condition. The first effect of the overt rehearsal procedure is on immediate recall scores. Recall levels were greater with silent than with overt rehearsal for the first three probe positions, but this difference was reversed at the fourth position. These data were analyzed with a 2 (rehearsal condition) by 5 (probe position) analysis of variance, with each S's score being the total correct recalls over five replications. In addition to the usual large effect of probe position, the interaction was also significant: $F(4,248)=4.49, p<.01$.

The final recall data show first of all a clear replication of the negative recency effect in all four classifications (for both types of rehearsal and for previously tested and untested items). These data pose some problems of analysis because of the very low recall rates for some of the probe positions. Accordingly, scores for the two types of test items (tested and untested) were pooled for each $\mathrm{S}$ and analyzed with the same classification used for the immediate recall scores. The probe position effect (the decline in recall over positions) was significant: $F(4,248)=$ 20.52, $\mathrm{p}<.001$; and the interaction of rehearsal condition and probe position was again significant: $\mathrm{F}(4,248)=2.51, \mathrm{p}<.05$.

Far from improving final recall levels or equating recall over probe positions, the overt rehearsal procedure

Table 1

Immediate (T1) and Final (T2) Recall for Silent and Overt Rehearsal

\begin{tabular}{ccccccc}
\hline & & \multicolumn{5}{c}{ Probe Position } \\
\cline { 3 - 7 } Rehearsal Condition & 1 & 2 & 3 & 4 & \multicolumn{1}{c}{5} \\
\hline \multirow{3}{*}{ Silent } & T1 & .46 & .41 & .39 & .49 & 1.00 \\
& T2-PT* & .27 & .26 & .19 & .09 & .07 \\
& T2-NPT** & .11 & .10 & .12 & .07 & .03 \\
& T1 & .34 & .33 & .27 & .61 & 1.00 \\
Overt & T2-PT & .17 & .19 & .12 & .12 & .01 \\
& T2-NPT & .10 & .06 & .07 & .09 & .05 \\
\hline
\end{tabular}

*Previously tested in immediate recall

**Not previously tested in immediate recall 
applied to PA lists seems to reproduce the negative recency effect obtained under silent rehearsal conditions, but at lower levels of recall. It also has the effect of depressing immediate recall levels. This latter effect is not a new one; Tzeng et al (1972) report a similar finding for free recall, and Murdock (1967) shows the effects of verbalization (overt rehearsal) and nonverbalization on PA probe tests that suggest the same crossover effect that was obtained here (see Figs. 1 and 3 in that article).

Whether a more extensive overt rehearsal procedure would succeed in reducing negative recency effects remains problematic; and it is not completely clear that this kind of overt rehearsal requirement does, in fact, preclude cumulative rehearsal of prior pairs. In any event, a simple overt rehearsal of paired associates is not accomplishing as much as its covert counterpart does in producing accessible material in memory. The negative recency effect with paired associates could not reflect a simple absence of "attention" to the final pairs in a list, given the rehearsal task and its failure to eliminate these effects. Apart from reference to the generation of mediators, or mnemonics, or to coding processes in general, it seems quite difficult to specify with any precision the nature of the processing required to produce long-term retention of associations. As Craik \& Lockhart (1972) suggest, the production of stable long-term memories is not simply a matter of the amount of processing, at least when this is scaled in terms of number of rote (literal) repetitions of to-be-remembered material; instead, the type or "depth" of processing must be considered as well.

\section{REFERENCES}

Atkinson, R. C., \& Shiffrin, R. M. The control of short-term memory. Scientific American, 1971, 225, 82-90.

Craik, F. I. M., \& Lockhart, R. S. Levels of processing: A framework for memory research. Journal of Verbal Learning \& Verbal Behavior, 1972, 11, 671-684.

Madigan, S. A., \& McCabe, L. Perfect recall and total forgetting: A problem for models of short-term memory. Journal of Verbal Learning \& Verbal Behavior, 1971, 10, 101-106.

Murdock, B. B., Jr. Auditory and visual stores in short-term memory. Acta Psychologica, 1967, 27, 316-324.

$R$ undus, $D$. Analysis of the rehearsal process in free recall. Journal of Experimental Psychology, 1971, 89, 63-77.

Tzeng, O. J., Snyder, H. I., \& Hung, D. C. The effects of overt intralist retrieval of subsequent free recall. Psychonomic Science, 1972, 28, 103-105.

(Received for publication March 21, 1973.) 